\title{
Fast 3D Shape Recovery from Shadows Projected on Arbitrary Curved Surfaces
}

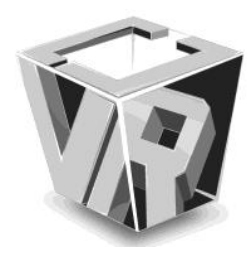

\author{
Yukihiro Yamashita, Fumihiko Sakaue and Jun Sato
}

\begin{abstract}
Department of Computer Science and Engineering, Nagoya Institute of Technology
\end{abstract}
Nagoya 466-8555, Japan

\begin{abstract}
The shadow based 3D surface reconstruction methods usually assume that shadows are projected on planar surfaces. However, shadows are often projected on curved surfaces in the real scene. Recently, the shadow graph has been proposed for representing shadow information efficiently, and for recovering 3D shapes from shadows projected on curved surfaces. Unfortunately, the method requires a large computational cost and is weak to the image intensity noises. In this paper, we introduce 1D shadow graphs which can represent shadow information quite efficiently, and can be used for recovering 3D shapes with much smaller computational costs than before. We also extend our method, so that we can recover 3D shape quite accurately by using shading information as well as shadow information. The proposed method is tested by using the real and synthetic images.
\end{abstract}

Index Terms - 3D reconstruction, shadow graph, shape from shadow.

\section{INTRODUCTION}

The shape from shading has been studied extensively for recovering accurate $3 \mathrm{D}$ shapes, and the propagation based methods [1], the energy minimization methods [2] and the local methods [3] have been proposed. Although the shape from shading is useful for recovering 3D shapes from single view images, it requires strong constraints such as smoothness of surfaces, constant albedo, and so on. It is also weak to image noises, since it uses differential properties of surface and intensity.

If the light source moves in the 3D space, shadows of objects caused by the light are also useful for recovering 3D shapes. Such methods are called shape from shadow [4, 5]. Recently, the shadow graph [5] has been proposed for representing shadow information in images. It can represent the relationship between attached shadows and cast shadows in a directed graph with costs, and is used for recovering 3D shapes from shortest path finding. Unfortunately, shortest path finding in the shadow graph requires a lot of computational costs, and we cannot recover 3D shapes in a reasonable amount of time.

To cope with this problem, we in this paper introduce 1D shadow graphs which are extracted from constant circular motions of a light source. We show that the 1D shadow graphs

Manuscript received on 16 March 2009

E-Mail: junsato@nitech.ac.jp can be used efficiently for recovering 3D shapes from shadow information, and the computational costs can be reduced drastically. We also show that shading information can be used for extracting accurate shadow areas, and hence for recovering accurate 3D shapes from shadows.

\section{SHADOW GRAPH}

We first explain the shadow graph proposed by Yu et al [5]. The shadow graph is a directed graph with costs which represent the relative height of points computed from the shadow and the orientation of light source. Fig.1 (b) is a shadow graph of Fig.1 (a). As shown in these figures, a node of the graph corresponds to a point on the surface, and the cost between two nodes represents the relative height between two points on the surface.

Let us consider a 3D surface as shown in Fig.1 (c), and let $h(x)$ be the height of the surface at point $x$. The light source is at infinity and the orientation of the light source is $\theta$. Suppose [ $x_{\mathrm{a}}$, $x_{\mathrm{c}}$ ] is a shadow area as shown in Fig.1 (c). $x_{\mathrm{a}}$ is the light source side boundary of the shadow area, and is called an attached shadow boundary. $x_{\mathrm{c}}$ is the anti-light source side boundary of the shadow area, and is called a cast shadow boundary. In the shadow graph, the following two types of shadow constraints are extracted from the attached and the cast shadow boundaries, and these constraints are represented by the cost of edges of the graph.

\subsection{Shadow Constraints}

We first consider constraints for a surface point in the shadow area $\left[x_{a}, x_{c}\right]$. Let us consider a point $x_{s}$ which is in the shadow area $\left[x_{a}, x_{c}\right]$. as shown in Fig.1 (c). Since $x_{s}$ is in the shadow area, it must be below the light line $L$ to the point $x_{a}$. Thus, $h\left(x_{s}\right)$ is smaller than $h\left(x_{a}\right)-\left(x_{a}-x\right) \tan \theta$. This means the following inequality holds for any point $x$ in the shadow area $\left[x_{a}, x_{c}\right]$ :

$h(x) \leq h\left(x_{a}\right)-\left(x_{a}-x\right) \tan \theta$

where, $\theta \neq 90^{\circ}$. Equation (1) is called the shadow constraints. The shadow constraints exist between an attached shadow boundary point $x_{a}$ and any point $x$ in the shadow area $\left[x_{a}, x_{c}\right]$. The shadow constraints are represented by a directed graph, in 
which the point $x_{a}$ and $x$ are represented by nodes, and $-\left(x_{a}-x\right) \tan \theta$ is represented by the cost $C_{x}^{x_{a}}$ between node $x_{a}$ and node $x$ as shown in Fig.1.

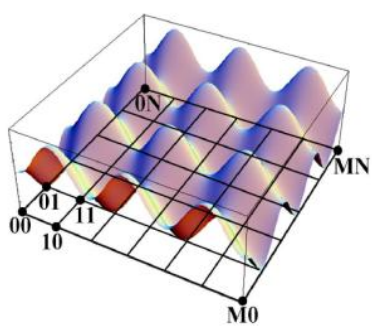

(a) 3D surface

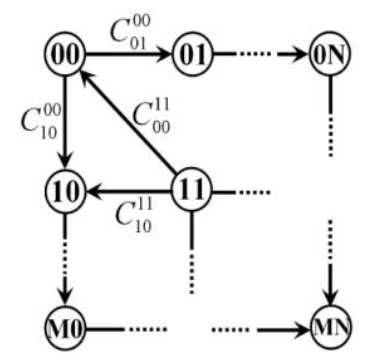

(b) shadow graph

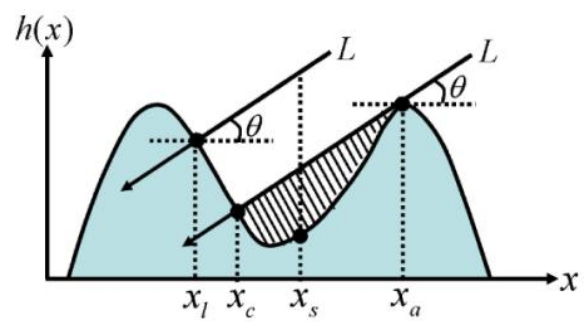

(c) shadow and anti-shadow constraints

Fig.1. Shadow graph and constraints. (a) shows an example 3D surface and (b) shows a shadow graph representation of shadow information. $C_{y}^{x}$ is a cost between node $x$ and node $y$ (c) shows shadow constraints and anti-shadow constraints. The point $x_{s}$ in the shadow area $\left[x_{a}, x_{c}\right]$ must be below the light line $L$ which goes through the attached shadow boundary $x_{a}$ (shadow constraints). Also, the point $x_{s}$ must be below the light line $L$ which goes through $x_{l}$ (anti-shadow constraints).

\subsection{Anti-Shadow Constraints}

We next consider constraints for a surface point which is not in the shadow area $\left[x_{a}, x_{c}\right]$. Let us consider a point $x_{l}$ which is not in the shadow area $\left[x_{a}, x_{c}\right]$ as shown in Fig.1 (c). Then, any point $x$ which is in the light source side of $x_{l}$ must be below the light line $L$ to the point $x_{l}$. Thus, the following inequality holds for any point $x$ in the light source side of $x_{l}$, even if $x$ is in a shadow area:

$$
h(x) \leq h\left(x_{l}\right)+\left(x-x_{l}\right) \tan \theta
$$

where, $\theta \neq 90^{\circ}$. (2) is called the anti-shadow constraints. The anti-shadow constraints are also represented by a directed graph, in which $\left(x-x_{l}\right) \tan \theta$ is the cost $C_{x}^{x_{l}}$ between node $x_{l}$ and node $x$.

\subsection{D Shape Recovery from Shadow Graph}

Suppose a 3D surface is illuminated by a moving light source at infinity, and is viewed from a fixed camera with orthogonal projection. In the shadow graph extracted from different orientations of light source, each node is a pixel of the camera image, and each cost of edge between a pair of nodes represents a relative height between a pair of pixels. Thus, the upper bound of relative height between an arbitrary pair of image pixels can be computed by finding the shortest path between a pair of nodes in the shadow graph. Thus, the 3D shape can be recovered by finding the shortest paths for all the pairs of nodes in the graph.

There are many methods for finding the shortest path in a graph. However, most of them do not work properly when there are negative costs in a graph as the shadow graph. Thus, in this research we use Warshall-Floyd algorithm [6] which can cope with negative costs.

If we find all the shortest paths between a fixed point and other points in the graph, then we can recover relative height between the fixed point and other points, and hence we can recover the $3 \mathrm{D}$ shape relative to the fixed point. Unfortunately, this method requires a lot of computational cost, and the cost for an $N \times N$ image is the order of $O\left(N^{6}\right)$, since the Warshall-Floyd algorithm requires $O\left(N^{3}\right)$ for $N$ nodes, and we have $N^{2}$ nodes from an $N \times N$ image. This is unrealistic in practice.

\section{FAST 3D SHAPE RECOVERY FROM 1D SHADOW GRAPHS}

In this section, we propose a method for recovering the $3 \mathrm{D}$ shape from a combination of 1D shadow graphs. In this method, we consider a single 2D image as a set of 1D image data in two independent directions, such as row and column directions, and generate 1D shadow graphs for these 1D image data. These 1D shadow graphs are combined together for recovering the $3 \mathrm{D}$ shape of the surface. Since the number of nodes used in a single path finding in a $1 \mathrm{D}$ shadow graph is very small, the computational cost for recovering 3D shapes is much smaller than the original method. The computational cost for $N \times N$ image is $O\left(N^{4}\right)$ in this method, while the original method requires $O\left(N^{6}\right)$.

In the following part of this section, we explain our method assuming these two independent directions are row and column directions. However, the method can be applied for any two independent directions of light source motions.
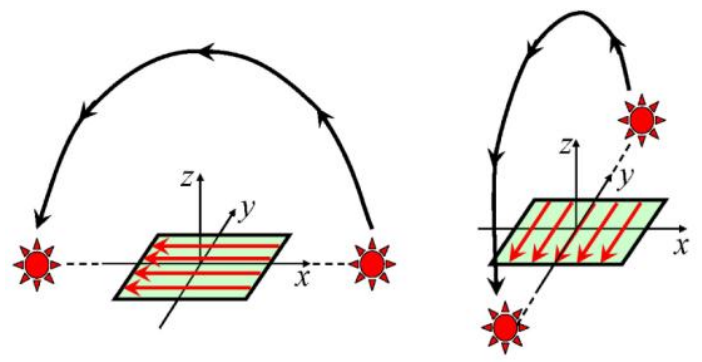

Fig. 2. Two independent motions of a light source at infinity. 


\subsection{D Slice Reconstruction from 1D Shadow Graph}

Suppose the light source moves in row and column directions in the 3D space as shown in Fig.2. Under these light source motions, the extracted shadow graphs are connected only in the row direction or in the column direction, and thus the extracted graphs are independent in rows and columns of an image. By finding the shortest path in each $1 \mathrm{D}$ graph, we can recover a set of row slices and column slices of the 3D surface with low computational cost. Since 1D graphs do not have connection with other rows and columns, the row and column slices of the surface recovered from the 1D graph have an ambiguity of vertical translation. Thus, we next fix the vertical position of each slice and recover the whole shape of the surface.
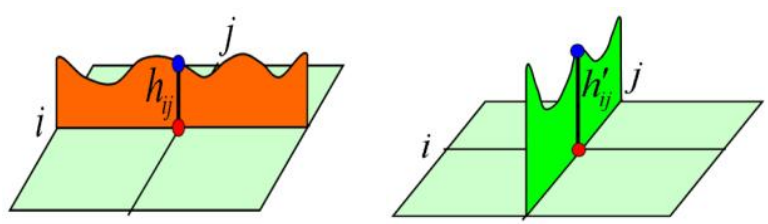

Fig. 3. The row slice and the column slice recovered from 1D shadow graphs. The pixel $(i, j)$ has height $h_{i j}$ in the $i$ th row slice, and has height $h_{i j}^{\prime}$ in the $j$ th column slice.

\subsection{D Shape Recovery from 1D Shadow Graph}

Let us consider a pixel $(i, j)$ in the $N \times N$ image. From the $3 \mathrm{D}$ slice reconstruction described in the previous section, we have two slices which go through the pixel $(i, j)$, that is $i$ th row slice and $j$ th column slice as shown in Fig.3. Since each slice reconstructed from 1D Shadow Graph is independent of the other slices, each slice has ambiguity of vertical translation. Although each slice has ambiguity of vertical translation, the height $h_{i j}$ of pixel $(i, j)$ in the $i$ th row slice must be identical to the height $h_{i j}^{\prime}$ of pixel $(i, j)$ in the $j$ th column slice, if there is no error. Thus, we consider the following cost function for all the points in the image.

$$
\varepsilon=\sum_{i, j}\left(h_{i j}+t_{i}-h_{i j}^{\prime}-t_{j}^{\prime}\right)^{2}
$$

where, $t_{i}$ is a vertical translation of $i$ th row slice, and $t_{j}^{\prime}$ is that of $j$ th column slice. By computing $t_{i}(i=1, \ldots, N)$ and $t_{j}^{\prime}$ $(j=1, \ldots, N)$ which minimize $\varepsilon$ in (3), we can recover the 3D shape of the surface, which minimizes the error in vertical position of row and column slices. However, the row and column slices recovered from 1D shadow graphs sometimes include large errors caused by image noises and missing edges in the shadow graph. Thus, we in this paper apply a robust regression algorithm, RANSAC [7], for combining row and column slices accurately excluding outliers in the slice data.

Suppose we have $N$ column slices and $N$ row slices from an $N \times N$ image. These row slices and column slices intersect at $N^{2}$ intersections in the combined data. These slice intersections sometimes include outliers, where the height of row slice and/or column slice has a large error. These slice intersections should be excluded from the minimization of (3).

For excluding these outliers, we first combine row slices and column slices one by one, so that a row slice and a column slice have identical height, $h_{i j}=h_{i j}^{\prime}$, at the knot $(i, j)$ of these two slices, as shown in Fig.4. Then, $2 N-1$ knots are enough for fixing relative height of $N$ row slices and $N$ column slices. Thus, we chose a set of $2 N-1$ knots randomly, and count the number of intersections where the relative height between row slice and column slice is less than a threshold. We iterate the procedure several times, and find a set of knots which provides us the maximum number of inlier intersections. Then, $t_{i}(i=1, \ldots, N)$ and $t_{j}^{\prime}(j=1, \ldots, N)$ are computed by minimizing (3) from all the intersections except outliers. Finally the 3D shape of the surface is recovered by translating the $i$ th row slice with $t_{i}$ and the $j$ th column slice with $t_{j}^{\prime}$.

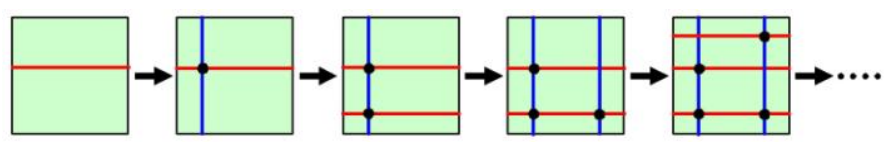

Fig. 4. The combination of row slices and column slices. The horizontal and vertical lines represent selected row and column slices, and the black dots show knots of two slices where the height of two slices is identical. The relative height of $N$ row slices and $N$ column slices can be fixed from $2 N-1$ knots.

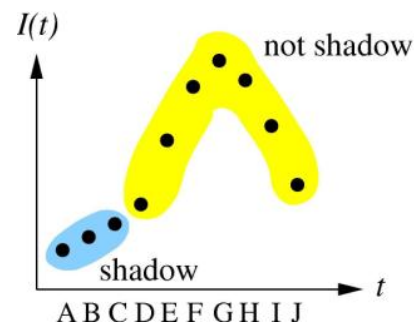

(a) intensity history with Inter-reflections

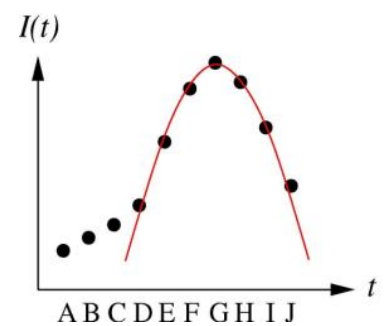

(b) quadratic curve fitting by RANSAC
Fig. 5. The shadow extraction from intensity history. (a) and (b) show intensity history of a fixed point obtained by moving the light source. The horizontal axis corresponds to the light source position, and the vertical axis shows the intensity at the fixed point. The point is in the shadow area when the light source position is A, B and C. However, its intensity is not zero because of inter-reflections. (b) shows a quadratic curve fitted to the intensity history by using RANSAC.

\section{EXTRACTING SHADOWS FROM SHADING INFORMATION}

In general shadow areas are extracted from images by using a threshold on image intensity. However, a single threshold is not enough for separating shadow areas from others properly. Furthermore, inter-reflections among surfaces cause distortions in surface brightness, and shadow areas are often classified into non-shadow areas because of the inter-reflections. Thus, we in this section use shading information, i.e. intensity history [8] of image points, for extracting shadow areas properly.

The intensity history of an image point is a set of image 
intensities obtained by changing the light source position. Suppose the light at infinity moves with a constant angular velocity. Then, the image intensity at a fixed image point changes sinusoidally. However, because of the inter-reflections, the intensity history is often distorted as shown in Fig.5 (a). Thus, we fit a quadratic curve to the intensity history by using RANSAC as shown in Fig.5 (b), and classify an image point into shadow area if the intensity of the point is outlier to the quadratic curve. By using the shadow areas extracted from the intensity history, we can recover 3D shapes accurately and reliably from 1D shadow graphs proposed in section 3 .

\section{REFINING SHDOW GRAPHS FROM SHADING INFORMATION}

The intensity history can also be used for refining shadow graphs. The shadow graph sometimes has incorrect edges because of the errors in shadow boundaries. In particular, attached shadow boundaries are sometimes unstable, since they are surface points where light lines are tangent to the surface.

Suppose we have a 3D surface whose row or column slice is as shown in Fig. 6 (a). If we have a correct attached shadow boundary, we can reconstruct the slice correctly. However, if the attached shadow boundary has some error, the reconstructed shape of the slice is distorted as shown in Fig. 6 (b). This is because a wrong edge is made from the wrong attached shadow boundary in the shadow graph.

Fortunately, such wrong edges can be removed by using the intensity history. By using the intensity history, we can roughly guess the surface orientation at each point on the surface. This is achieved by simply finding a light source orientation which provides us maximum intensity in the intensity history curve. Such orientation of the light source coincides with the orientation of the surface at each point in the slice. Although there exists one dimensional ambiguity in the measured surface orientation, the ambiguity is in the direction perpendicular to the slice and does not cause any problem in the following discussions.

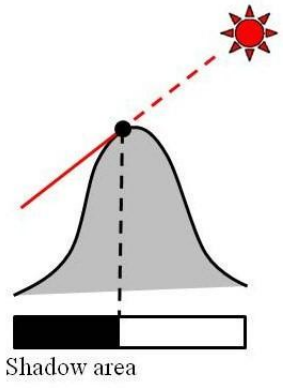

(a)

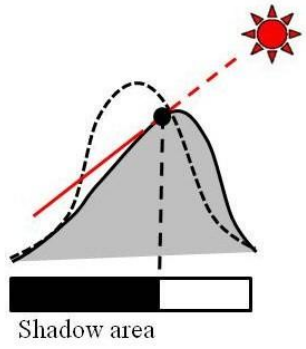

(b)
Fig. 6. Shape distortion caused by the error in attached shadow boundaries.

In the slice, the surface orientation at an attached shadow boundary is perpendicular to the light orientation. Thus, if the surface orientation of an attached shadow boundary computed from the light orientation is far from the surface orientation computed from the intensity history, it is considered as a wrong attached shadow boundary. We remove edges in the shadow graph, whose nodes correspond to such wrong attached shadow boundaries. By removing these wrong edges in the 1D shadow graphs, we can refine the $3 \mathrm{D}$ shapes.

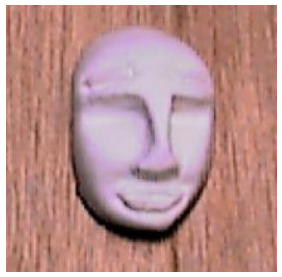

(a) face model

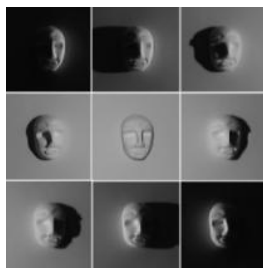

(b) example images

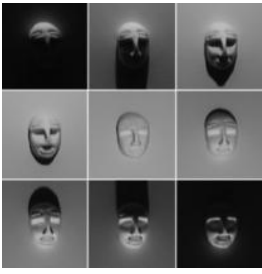

(c) example images
Fig. 7. The face model and its images used in our experiment.

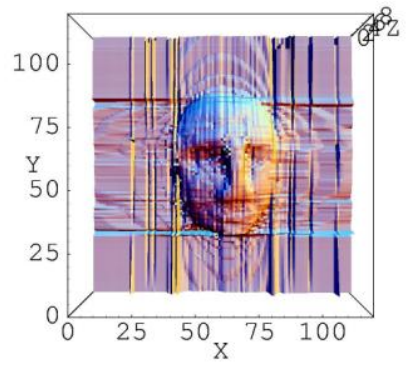

(a)

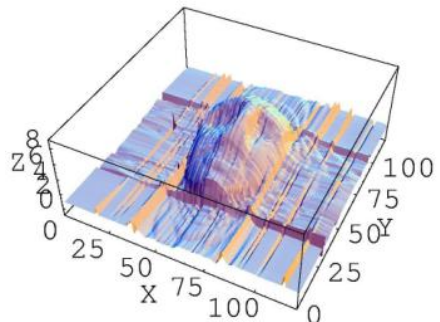

(b)
Fig. 8. The 3D shape recovered from the proposed method.

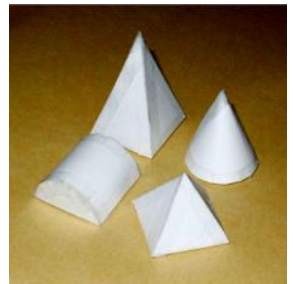

(a)

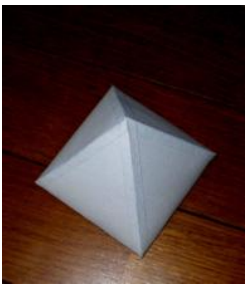

(c)

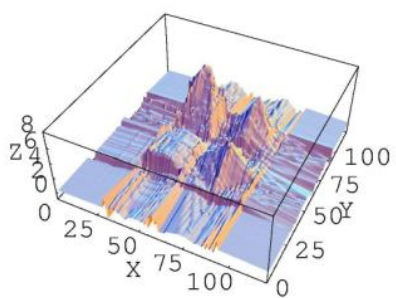

(b)

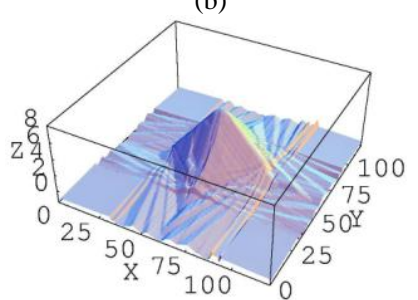

(d)
Fig.9. The 3D shape recovered from the proposed method (other objects).

\section{EXPERIMENTS}

\subsection{Real Image Experiments}

In this section, we show the results from 3D surface reconstruction from real images. Fig.7 (a) shows a face model used in this experiment. The camera is fixed in front of this object, and the light is moved around the object. The motion of the light source is circular with the radius of $1 \mathrm{~m}$. It moves from 0 degrees to 180 degrees with the interval of 10 degrees in two directions, one in the row direction and the other in the column direction of the camera image. Hence, we obtained 38 images in total. These image are shown in Fig.7 (b) and (c). The size of 
each image is $120 \times 120$. As shown in Fig.7, shadows are on the complex curved surface of the face and we have very complex shadows. The proposed method is applied to these images and the 3D shape of the face is recovered. The recovered 3D shape is shown in Fig.8. Fig.8 (a) and (b) are the result viewed from two different viewpoints. As shown in Fig.8, the complex shape of the face is recovered properly, and we find the proposed method is very efficient for recovering arbitrary $3 \mathrm{D}$ surfaces from shadows. The results from some other 3D objects are shown in Fig.9.

\subsection{Computational Cost Evaluation}

We next evaluate the computational cost of the proposed method. The computational time of the proposed method was measured changing the image size from $30 \times 30$ to $120 \times 120$, and was compared with that of the 2D shadow graph method.

Fig. 10 shows the computational time of these two methods. As shown in this figure, the computational time of the proposed method is much faster than that of the 2D shadow graph method.

\subsection{Accuracy Evaluation}

We next evaluate the accuracy of the proposed method by using synthetic image data.

In this experiment, we used a sinusoidal surface shown in Fig.11 (a). The camera is fixed in front of the object assuming orthogonal projection. The light source is at the infinity and moves around the object from 0 degrees to 180 degrees in two directions, one in the direction of rows and the other in the direction of columns of camera image. The image size is $120 \times 120$. The accuracy of the proposed method is evaluated by the difference $D$ between the true height $h_{i j}$ and the height $h_{i j}^{\prime}$ recovered from the proposed method as follows:

$$
D=\frac{1}{N^{2}} \sum_{i, j} \frac{\left\|h_{i j}-h_{i j}^{\prime}\right\|}{h_{\max }-h_{\min }}
$$

where, $h_{\max }$ and $h_{\min }$ are the maximum height and the minimum height of the true shape, and $N$ is the size of the image.

1) Light Source Interval and Accuracy of Reconstruction: We first evaluated the relationship between the interval of light source motions and the accuracy of reconstruction. We evaluated in four different intervals of light source motions, that is $5^{\circ}, 10^{\circ}, 15^{\circ}$ and $20^{\circ}$, in which the number of images is 74,38 , 26 and 20 respectively. We added intensity noises with the standard deviation of 2 to all the image pixels. There is no inter-reflection in this experiment.

The 3D shapes recovered from the proposed method in these four sampling intervals are shown in Fig.11, and the relationship between the sampling interval and the error $D$ in (4) is shown in Fig.12. As shown in these figures, the accuracy of the proposed method degrades as the number of light source motions goes down. However, we can obtain reasonable shapes from the proposed method, even though the number of light source motions is small.

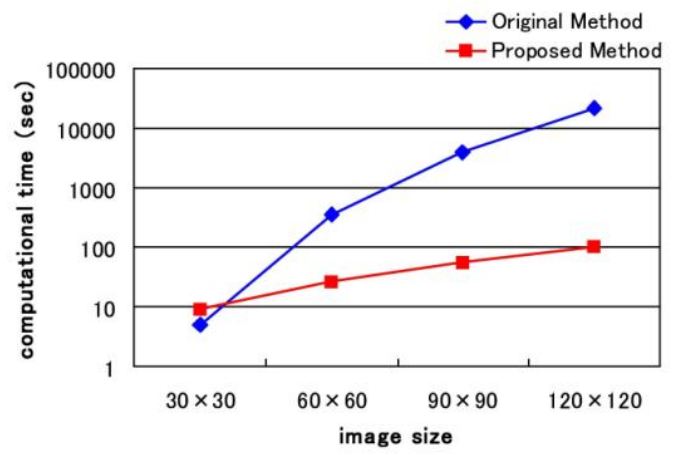

Fig. 10. The comparison of computational time. The computational time of the proposed method is compared with that of the $2 \mathrm{D}$ shadow graph method (original method) changing the image size from $30 \times 30$ to $120 \times 120$.

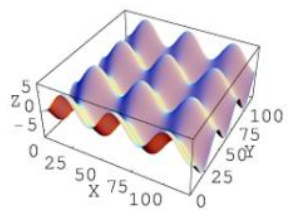

(a)

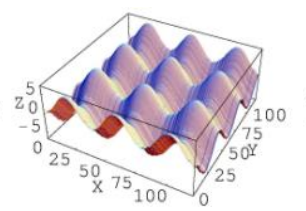

(b) $5^{\circ}$

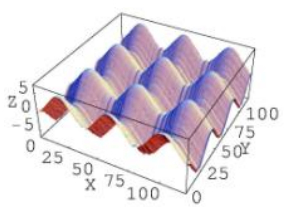

(c) $10^{\circ}$

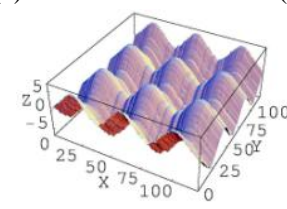

(d) $15^{\circ}$

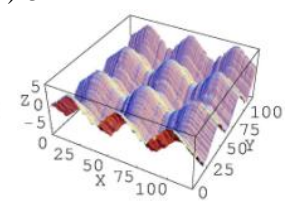

(e) $20^{\circ}$
Fig.11. The interval of light source motions and the 3D shape recovered from the proposed method. (a) shows a 3D surface used in this experiment. (b), (c), (d) and (e) show the 3D shapes recovered from light source interval of $5^{\circ}, 10^{\circ}$, $15^{\circ}$ and $20^{\circ}$ respectively.

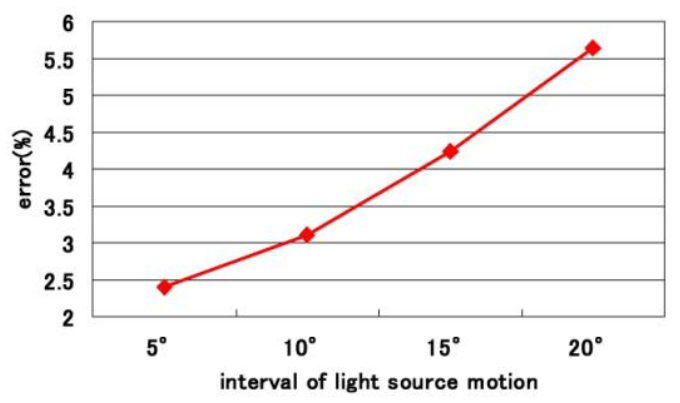

Fig. 12. The relationship between the interval of light source motions and the error $D$ of recovered shape.

2) Inter-Reflections and Accuracy of Reconstruction: We next evaluated the tolerance of the proposed method to the intensity distortions caused by inter-reflections. We generated synthetic images which include inter-reflections up to 3rd order, and used them for recovering 3D shapes from the proposed method. The 3D object is the same as before. The interval of light source motions is $10^{\circ}$, and the intensity noise with the standard deviation of 2 is added to all the image pixels.

The 3D shape reconstructed from the proposed method is shown in Fig.13 (a). As shown in this figure, the 3D shape is recovered quite accurately, even if we have inter-reflections in 
images. For comparison we recovered the same object without using intensity history for extracting shadow areas. The result is shown in Fig.13 (b). As shown in Fig.13 (a) and (b), the intensity history is very useful for recovering accurate $3 \mathrm{D}$ shape from shadows. The error $D$ of recovered shape is $4.93 \%$ in (a) and $16.43 \%$ in (b) respectively. Fig.14 (a) shows an example image with strong inter-reflections, and (b) and (c) show shadow areas extracted by using a single threshold and the intensity history respectively. As shown in these figures, the proposed method works quite well under inter-reflections.

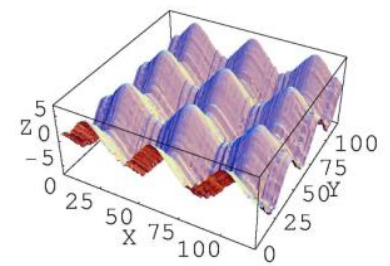

(a) using intensity history

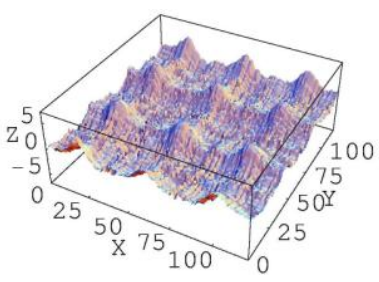

(b) using a single threshold
Fig. 13. The inter-reflections and the accuracy of 3D recovery. (a) shows a 3D shape recovered from shadows extracted by using the intensity history. (b) shows that recovered from a single threshold.

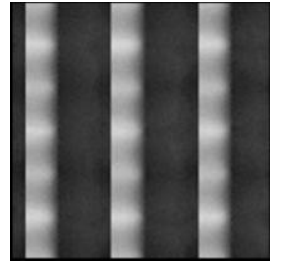

(a) example image

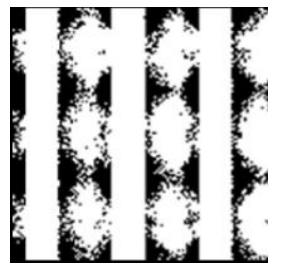

(b) single threshold

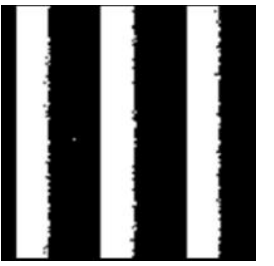

(c) intensity history
Fig. 14. The separation of shadow and non-shadow areas. (a) shows an example intensity image. The black areas in (b) show shadow areas obtained from a single threshold, while the black areas in (c) show those obtained from the intensity history.

\section{CONCLUSION}

In this paper, we proposed a method for recovering 3D shapes from shadow information projected onto arbitrary curved surfaces. We showed that 1D shadow graphs obtained from two independent orbits of light source motions can be used efficiently for recovering accurate 3D shapes from shadows with small computational costs. We also showed that intensity history is very useful for extracting accurate shadow areas and for recovering accurate 3D shapes from shadow information. The proposed method is robust to intensity noises and is very practical, since light source motions are often circular motions, such as sun motions.

The future work includes the extension of the proposed method, so that it can recover 3D shapes from unknown light source motions.

\section{REFERENCES}

[1] B.K.P. Horn, Shape from Shading: A Method for Obtaining the Shape of a Smooth Opaque Object from One View, PhD thesis, MIT, 1970.

[2] K. Ikeuchi, B.K.P. Horn, Numerical Shape from Shading and Occluding Boundaries, Artificial Intelligence, vol.17, no.1-3, pp.141-184, 1981.
[3] A.P. Pentland, Local Shading Analysis, Proc. IEEE Transactions on Pattern Analysis and Machine Intelligence, vol.6, pp.170-187, 1984.

[4] M. Daum and G. Dudek, On 3-D Surface Reconstruction using Shape from Shadows, Proc. Conference on Computer Vision and Pattern Recognition, 1998.

[5] Y. Yu, J.T. Chang, Shadow Graphs and 3D Texture Reconstruction, International Journal of Computer Vision, vol.62, no.1-2, pp.35-60, 2005.

[6] T. Cormen, C. Leiserson, R. Rivest, Introduction to Algorithms, MIT Press, 1990

[7] M.A. Fischler and R.C. Bolles, Random Sample Consensus: A Paradigm for Model Fitting with Applications to Image Analysis and Automated Cartography, Communications of the ACM., Vol.24, No.6, pp.381-395, 1981.

[8] T. Okabe, I. Sato, Q. Yu, Y. Sato, Shape Recovery based on Similarity in Radiance Changes under Varying Illumination, Proc. MIRU, OS-A8-02, pp.333-339, 2007.

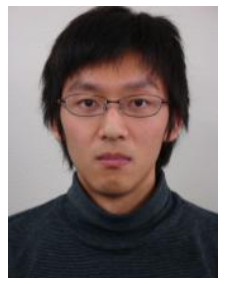

Yukihiro Yamashita received B.E. degree from the Department of Computer Science, Nagoya Institute of Technology, Japan in 2009. He is currently a master course student at the Department of Computer Science and Engineering, Graduate School of Engineering, Nagoya Institute of Technology.

His research interests include computer vision, 3D reconstruction and photometry.

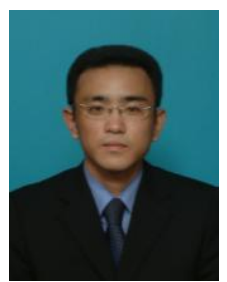

Fumihiko Sakaue received the B.E., M.E. and Ph D. degrees in Information technology from Okayama University, Japan in 2001, 2003 and 2006, respectively. $\mathrm{He}$ is currently an assistant professor at the Department of Computer Science and Engineering, Nagoya Institute of Technology.

His research interests include computer vision, photometric image analysis, pattern recognition and mixed reality.

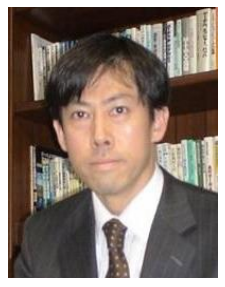

Jun Sato received B.E. degree from Nagoya Institute of Technology, Japan in 1984, and Ph.D. degree in information engineering from the University of Cambridge, England in 1997. From 1996 to 1998 he was a research associate at the Department of Engineering, University of Cambridge. During 1997--1998, he was an invited researcher at ATR Human Information Processing Research Laboratories, Kyoto. He joined the Department of Electrical and Computer Engineering, Nagoya Institute of Technology in 1998 as an associate professor. He is currently a professor at the Department of Computer Science and Engineering, Nagoya Institute of Technology.

His research interests include computer vision, geometric invariants, visual navigation, visual interface and mixed reality. He was awarded the Best Science Paper Prize of BMVC twice in 1994 and 1997, the Best Paper Prize of SSII'99 and the Special Session Prize of MIRU'07, etc. He is a member of the IEEE, IEICE and IPSJ. 